\title{
Edge Semientire Block Graph
}

\author{
Venkanagouda M. Goudar \\ Department of Mathematics \\ Siddhartha Institute of Technology, Affiliated to \\ SSAHE, Tumkur, Karnataka, India 572105
}

\author{
Jagadeesh $\mathrm{N}$ \\ Sri Goutam Research center \\ Siddhartha Institute of Technology, Tumkur, \\ Affiliated to SSAHE. \\ Kalpataru first grade Science College,Tiptur, \\ Karnataka, India.
}

\begin{abstract}
In this communications, the edge semientire block graph of a graph is introduced. We present a characterization of those graphs whose edge semientire block graph is planar, outer planar, Eulerian, Hamiltonian with crossing number one.
\end{abstract}

\section{General Terms}

Semientire graph, Line graph.

\section{Keywords}

Edge Semientire graph, line graph, inner vertex number,

Mathematics Subject Classification: 10C 75, 10C 10.

\section{INTRODUCTION}

The concept of block edge cut vertex graph was introduced by Venkanagouda M Goudar [5]. For the graph $G(p, q)$, if $B=u_{1}$, $u_{2}, \ldots u_{r}: r \geq 2$ is a block of $G$, then we say that the vertex $u_{i}$ and the block $\mathrm{B}$ are incident with each other. If two blocks $\mathrm{B}_{1}$ and $\mathrm{B}_{2}$ are incident with a common cut vertex, then they are adjacent blocks. All undefined terminology will conform with that in Harary[2]. All graphs considered here are finite, undirected, planar and without loops or multiple edges. We now define the edge semientire block graph.

The Edge semientire block graph of a graph $\mathrm{G}$ denoted by $E_{b}(G)$ is the graph whose vertex set is the union of set of edges, set of blocks and set of regions of $G$ in which two vertices are adjacent if and only if the corresponding edges of $\mathrm{G}$ are adjacent, the corresponding edges lies on the blocks, the corresponding edges lies on the region and the corresponding blocks are incident to a cut vertex. The graph $\mathrm{G}$ and its edge semientire graph $\mathrm{E}_{\mathrm{b}}(\mathrm{G})$ is depicted in the figure 1 .
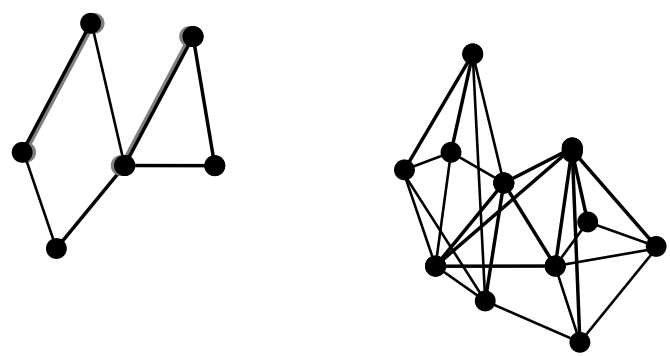

G

Figure 1.

$E_{b}(G)$.

The edgedegree of an edge $\{\mathrm{u} \mathrm{v}\}$ is the sum of the degree of the vertices of $\mathrm{u}$ and v. Blockdegree of a cut vertex is the number of blocks incident to the cut vertex and regionvertex is a vertex in $E_{b}(G)$ formed from the regions of $G$. The Blockvertex is a vertex in $E_{b}(G)$ formed from the block of $G$. For the planar graph $G$, the inner vertex number $i(G)$ of a graph $\mathrm{G}$ is the minimum number of vertices not belonging to the boundary of the exterior region in any embedding of $\mathrm{G}$ in the plane. The crossing number $\mathrm{Cr}(\mathrm{G})$ of a graph $\mathrm{G}$ is the minimum number of pair wise intersections of its edges when $\mathrm{G}$ is drawn in the plane. A graph $\mathrm{G}$ has crossing number $\mathrm{k}$ if A graph $G$ is outerplanar if $i(G)=0$ and graph $G$ is said to be minimally non-outerplanar if $i(G)=1$ as was given by Kulli [4].

\section{PRELIMINARY RESULTS}

We need the following results to prove further results.

Theorem 2.1[2]. If $G$ is a (p, q) graph whose vertices have degree $d_{i}$ then the line graph $L(G)$ has $q$ vertices and $q_{L}$ edges, where $q_{L}=-q+\frac{1}{2} \sum d_{i}^{2}$ edges.

Theorem 2.2 [2]. The line graph $\mathrm{L}(\mathrm{G})$ of a graph is planar if and only if $\mathrm{G}$ is planar, $\Delta \leq 4$ and if $\operatorname{deg} \mathrm{v}=4$ for a vertex $\mathrm{v}$ of $\mathrm{G}$, then $\mathrm{v}$ is a cut vertex.

Theorem 2.3[3]. A graph is planar if and only if it has no subgraph homeomorphic to $\mathrm{K}_{5}$ or $\mathrm{K}_{3,3}$.

Theorem 2.4 [4]. A graph is outerplanar if and only if it has no subgraph homeomorphic to $\mathrm{K}_{4}$ or $\mathrm{K}_{2,3}$.

Theorem 2.5 [3]. A finite graph $G$ is Eulerian if and only if all its vertex degrees are even.

\section{MAIN RESULTS}

Lemma 3.1: For any planar graph $\mathrm{G}, \mathrm{L}(\mathrm{G}) \subseteq \mathrm{E}_{\mathrm{b}}(\mathrm{G})$.

Theorem 3.2. For any planar graph $G, E_{b}(G)$ is always nonseparable.

Proof. We have the following cases.

Case 1. Suppose $\mathrm{G}$ be a tree $\mathrm{T}$. Let $\mathrm{e}_{1}, \mathrm{e}_{2}, \ldots \mathrm{e}_{\mathrm{n}}$ be the edges, $b_{1}=e_{1}, b_{2}=e_{2}, \ldots b_{n}=e_{n}$ be the blocks and $r_{1}$ be the region of $\mathrm{T}$. By the definition of line graph $\mathrm{L}(\mathrm{G}), \mathrm{e}_{1}, \mathrm{e}_{2}, \ldots \mathrm{e}_{\mathrm{n}}$ form a subgraph without isolated vertex. In $\mathrm{E}_{\mathrm{b}}(\mathrm{G})$, the regionvertex is adjacent to these vertices to form a graph without isolated vertex. Since there are $n$ blocks which are $K_{2}$, we have each $b_{1}$ $=\mathrm{e}_{1}, \mathrm{~b}_{2}=\mathrm{e}_{2}, \ldots \mathrm{b}_{\mathrm{n}}=\mathrm{e}_{\mathrm{n}}$ are adjacent to $\mathrm{e}_{1}, \mathrm{e}_{2}, \ldots \mathrm{e}_{\mathrm{n}}$. Further the blockvertices of $E_{b}(G)$ are adjacent without cut vertex. Hence $\mathrm{E}_{\mathrm{b}}(\mathrm{G})$ is nonseparable.

Case 2. Suppose a graph $G$ is contains at least two adjacent cut vertices and is non-tree. Let $e_{1}, e_{2}, \ldots e_{n} \in E(G), b_{1}=e_{1}, b_{2}$ $=e_{2}, \ldots b_{k}=e_{n}$ be the blocks and $r_{1}, r_{2}, \ldots r_{1}$ be the region of $G$. By the definition of line graph $\mathrm{L}(\mathrm{G})$, the edges incident to the cut vertices becomes a complete graph and $L(G)$ contains at least one cut vertex. By the definition of $E_{b}(G)$, the region vertices are adjacent to the vertices of $L(G)$ and the vertices of 
$\mathrm{L}(\mathrm{G})$ is adjacent to the blockvertices. Clearly $\mathrm{E}_{\mathrm{b}}(\mathrm{G})$ is nonseparable.

In the following theorem we obtain the number of vertices and edges of edge semientire block graph of a graph.

Theorem3.3 For any planar graph G, edge semientire block graph $E_{b}(G)$ whose vertices have degree $d_{i}$ has $(q+r+b)$ vertices and $\quad-q+\frac{1}{2} \sum d_{i}^{2}+\sum q_{j}+\sum \frac{b_{k}\left(b_{k}-1\right)}{2}+\sum q_{r}$ edges where $r$ the number of regions, $b$ the number of blocks $q_{j}$ the number edges in a block $b_{j}, b_{k}$ be the block degree of a cut vertex $C_{k}$ and $q_{r}$ be the number of edges region $r_{1}$.

Proof. By the definition of $E_{b}(G)$, the number of vertices is the union of edges, regions and blocks of $G$. Hence $E_{b}(G)$ has $(\mathrm{q}+\mathrm{r}+\mathrm{b})$ vertices. Further by the Theorem 2.1, number of edges in $\mathrm{L}(\mathrm{G})$ is $q_{L}=-q+\frac{1}{2} \sum d_{i}^{2}$. The degree of each regionvertex is the number of edges lies on that region in $\mathrm{G}$. Thus the number of edges in $E_{b}(G)$, is the sum of the number of edges in $\mathrm{L}(\mathrm{G})$, the number of edges bounded by the each region which is $\sum q_{r}$, number of edges lies on each block which is $\sum q_{j}$ and the number of edges in a complete graph $\mathrm{K}_{\mathrm{k}}$ which is obtained from each blockdegree and is $\frac{b_{k}\left(b_{k}-1\right)}{2}$. Henace the number of edges in $E_{b}(G)$ is

$$
-q+\frac{1}{2} \sum d_{i}^{2}+\sum q_{j}+\sum \quad \frac{b_{k}\left(b_{k}-1\right)}{2}+\sum q_{r}
$$

Theorem3.4 For any edge in a plane graph $G$ with edgedegree $\mathrm{n}$, the degree of a corresponding vertex in $\mathrm{E}_{\mathrm{b}}(\mathrm{G})$ is $\mathrm{i}$ ). $\mathrm{n}+1$ if edge lie on two regions and ii). $n$, otherwise.

Proof. Suppose an edge $e_{i} \in E(G)$ have degree $n$. We have the following cases.

Case1. Suppose the edge lie on two regions. Clearly (n-2) edges are adjacent to the edge $e_{i} \in E(G)$. By the definition of line graph, the degree of the vertex $e_{i} \in L(G)$ is $(n-2)$. By the definition of Edge semientire block graph, this edge is lies on two regions and lies on one block, hence degree of a vertex $e_{i} \in E(G)$ in $E_{b}(G)$, is $n-2+2+1=n+1$.

Case2. Further, if $e_{i} \in E_{b}(G)$, lie on one region then by the case 1 , the degree of a vertex $e_{I}$ in $E_{b}(G)$, is $n-2+1+1=n$.

In the following theorem we obtain the condition for the planarity on edge semientire block graph of a graph.

Theorem 3.5 For any planar graph $G$, the $E_{b}(G)$ is planar if and only if $G$ is i).a path $P_{n}$ or ii). a star $K_{1,3}$.

Proof. Suppose $E_{b}(G)$ is planar. We have the following cases.

Case 1.Assume that a graph $G$ is $K_{1,4}$. By the definition of line graph, $\mathrm{e}_{1}, \mathrm{e}_{2}, \mathrm{e}_{3}, \mathrm{e}_{4}$ form a complete graph $\mathrm{K}_{4}$ and it has one inner vertex. Since all edges $\mathrm{e}_{1}, \mathrm{e}_{2}, \mathrm{e}_{3}, \mathrm{e}_{4}$ lies on one region $r_{1}$, which is adjacent to all vertices of $K_{4}$ in $E_{b}(G)$. Clearly $E_{b}(G)$ is non-planar, a contradiction.

Case 2. Assume that a graph $\mathrm{G}$ is non -path and it is a tree with at least one sub graph as $\mathrm{K}_{1,3}$ and contains at least four edges. Clearly $\mathrm{L}\left(\mathrm{K}_{1,3}\right)=\mathrm{K}_{3}$. Also each edge is a block and all blocks are adjacent. So these blocks form a complete graph $\mathrm{K}_{3}$. Since all four edges lies on one region $r_{1}$ and are adjacent to all vertices of $K_{3}$ in $E_{b}(G)$. Clearly the fourth edge is also adjacent to $r_{1}$ and it crosses the edge already drawn, which is a contradiction.

Case 3. Assume that $\mathrm{G}$ is any graph which contains at least one block with three edges. Let $\mathrm{e}_{1}, \mathrm{e}_{2}, \mathrm{e}_{3}$ be the edges of a block $b_{i}$. These edges form one interior region $r_{j}$. By the definition of $E_{b}(G)$, the vertices $e_{1}, e_{2}, e_{3}, b_{i}, r_{j}$ forms graph homeomorphic to $\left\langle K_{5}\right\rangle$, which is non-planar, a contradiction.

By the Theorem 2.3, it is non planar, a contradiction.

Conversely, suppose $\mathrm{G}$ is a path. By the definition of $\mathrm{L}(\mathrm{G})$, $L\left(P_{n}\right)=P_{n-1}$. Since all edges all edges lies on only one region and each edge is a block then by definition of $E_{b}(G)$, it forms a graph homeomorphic to $\mathrm{K}_{4}$. Hence $\mathrm{E}_{\mathrm{b}}(\mathrm{G})$ is planar. Further if $\mathrm{G}$ is $\mathrm{K}_{1,3}$ then $\mathrm{L}\left(\mathrm{K}_{1,3}\right)=\mathrm{K}_{3}$. Since all three edges lies on only one region and each edge is a block then by definition of $E_{b}(G)$, it forms a graph homeomorphic to $K_{4}$. Hence $E_{b}(G)$ is planar.

In the following theorem we obtain the condition for the outer planarity on Edge semientire block graph of a graph.

Theorem 3.6 For any planar graph $G, E_{b}(G)$ is outer planar if and only if $G$ is a path $P_{n}$ for $n \leq 3$.

Proof. Suppose $E_{b}(G)$ is outer planar. Assume that $G$ is a path $P_{n} n \geq 4$. Suppose $n=4$. By the definition of line graph, $\mathrm{L}\left(\mathrm{P}_{4}\right)=\mathrm{P}_{3}$. Since all the edges $\mathrm{e}_{1}, \mathrm{e}_{2}, \mathrm{e}_{3}$ are lies on only one region $r_{1}$, then $E_{b}(G)$ forms $K_{4}-x$. Further each edge is a block, so each $e_{i}$ is adjacent to the blockvertex $b_{j}$. Lastly each blockvertex is adjacent to the corresponding edge to form $\mathrm{K}_{3}$. Clearly the non-pendent edge of $\mathrm{G}$ forms an inner vertex in $E_{b}(G)$, which is non- outerplanar, a contradiction. . Hence $G$ must be a path $P_{n}$ for $n \leq 3$.

Conversely, Suppose $G$ is a path $P_{n}$ for $n \leq 3$. Let $e_{1}, e_{2}, e_{3}$ be the edges in $G$. By the definition of line graph, $L\left[P_{n}\right]=P_{n-1}$. Further by the lemma $3.2, b_{1}=e_{1}, b_{2}=e_{2}, \ldots, b_{n-1}=e_{n-1}$ becomes n-1 pendant vertices and it becomes a caterpillar. Further the region vertex $r_{i}$ is adjacent to all the vertices of $L\left[P_{n}\right](G)$. Clearly $E_{b}(G)$ is outer planar.

In the following theorem we obtain the condition for the minimally non- outerplanar on Edge semientire block graph of a graph.

Theorem 3.7 For any planar graph $G, E_{b}(G)$ is minimally non-outer planar if and only if $\mathrm{G}$ is $\mathrm{P}_{4}$.

Proof. Suppose $E_{b}(G)$ is minimally non-outer planar. Assume that $G$ is a path $P_{n} n \geq 5$. Suppose $n=5$. By the definition of line graph, $\mathrm{L}\left(\mathrm{P}_{5}\right)=\mathrm{P}_{4}$. In a path, each edge is a block, for each $e_{i}$ is adjacent to $b_{i}$. Clearly the vertices of $L\left(P_{5}\right)$ and $b_{i}$ form a graph $\mathrm{C}_{4} \cdot \mathrm{C}_{4} \ldots \mathrm{C}_{4}$. Also the vertices $\mathrm{e}_{1}, \mathrm{e}_{2}, \mathrm{e}_{3}, \mathrm{e}_{4}$ are lies on only one region $r_{1}$, then by the definition of $E_{b}(G)$, It contains a graph with two inner vertex number, a contradiction. Hence $\mathrm{G}$ is a path $\mathrm{P}_{4}$

Conversely, Suppose $\mathrm{G}$ is a path $\mathrm{P}_{4}$. Let $\mathrm{e}_{1}, \mathrm{e}_{2}, \mathrm{e}_{3}$ be the edges in $\mathrm{G}$. By the definition of line graph, $\mathrm{L}\left[\mathrm{P}_{4}\right]=\mathrm{P}_{3}$. In a path, each edge is a block, for each $e_{i}$ is adjacent to $b_{i}$. Clearly the vertices of $\mathrm{L}\left(\mathrm{P}_{4}\right)$ and $\mathrm{b}_{\mathrm{i}}$ form a graph $\mathrm{C}_{4} \cdot \mathrm{C}_{4}$. In a tree all edges lies on only one region $r_{1}$ and this region is adjacent to all vertices of $L\left(P_{4}\right)$ in $E_{b}(G)$. the vertex $e_{3}$ which was the inner edge in $G$ becomes the inner vertex in $E_{b}(G)$. Clearly $E_{b}(G)$ is minimally non- outerplanar.

In the following theorem we obtain the condition for the crossing number one on Edge semientire block graph of a graph.

Theorem 3.8 For any planar graph $G, E_{b}(G)$ has crossing number one if and only if $\mathrm{G}$ is a tree with at least one vertex of degree two and an unique vertex of degree three.

Proof. Suppose $E_{b}(G)$ has crossing number one. We have the following cases. 
Case 1. Assume that a graph $G$ is a tree with two vertices $v_{i}$ and $\mathrm{v}_{\mathrm{j}}$ are of degree 3 . Let $\mathrm{e}_{\mathrm{i} 1}, \mathrm{e}_{\mathrm{i} 2}, \mathrm{e}_{\mathrm{i} 3}$ be the edges incident to the cut vertex $v_{i}$ and $e_{j 1}, e_{j 2}, e_{j 3}$ be the edges incident to $v_{j}$. By the definition of line graph, each star $v_{1}$ and $v_{2}$ form induced subgraph as $K_{4}$. In $E_{b}(G)$, the region vertex $r_{1}$ is adjacent to all vertices of $\mathrm{L}(\mathrm{G})$ to form a planar graph. Further the blockvertex is adjacent to the vertices of $L(G)$ those are the edges of block in $\mathrm{G}$ form graph homeomorphic to $\mathrm{K}_{5}$. Clearly $\mathrm{C}\left[\mathrm{E}_{\mathrm{b}}(\mathrm{G})\right] \geq 2$, a contradiction.

Case 2. Assume that a graph $\mathrm{G}$ is a tree with one cut vertex of degree 4. By the definition of line graph, $\mathrm{L}(\mathrm{G})$ form $\mathrm{K}_{4}$ as induced subgraph. Since each edge is a block, the vertices $e_{i}$ and $b_{i}$ for $i=1,2,3,4$ form $\mathrm{K}_{4} \mathrm{oK}_{1}$. Further the regionvertex is adjacent to all vertices of $L(G)$ such that $C\left[E_{b}(G)\right] \geq 2$, a contradiction. $\mathrm{G}$ is a tree with at least one vertex of degree two and an unique vertex of degree three.

Conversely suppose $\mathrm{G}$ is a tree with at least one vertex of degree two and an unique vertex of degree three. The line graph of a graph with a vertex of degree three becomes $\mathrm{K}_{3}$ as subgraph. Since the regionvertex is adjacent to all the vertices of a line graph, in $E_{b}(G)$, the line graph $L(G)$ and $a$ regionvertex forms a complete graph $\mathrm{K}_{4}$. Clearly it contains one inner vertex. By the definition block graph $B(G)$, all block vertices $b_{i}, b_{j}, b_{k}$ are adjacent to form $K_{3}$. Hence in $E_{b}(G)$ all block vertices becomes the inner vertices. By the condition $\mathrm{G}$ contains at least one vertex of degree two. The block vertex of this edge $b_{1}$ is adjacent to at least one vertex of $B(G)$. Clearly it crosses the edge we already drawn. Hence $G$ has crossing number one.

In the following theorem we obtain the condition for the non-

Eulerian on Edge semientire block graph of a graph.

Theorem 3.8 For any planar graph $G, E_{b}(G)$ is Eulerian if and only if the following conditions hold

i). Edgedegree of and edge is odd if the edge lies on two regions ii). Edgedegree of an edge is even if the edge lies on one region. iii). Each region contains even number of edges.

Proof. Suppose G is Eulerian. We have the following cases.

Case 1. Assume that edge lies on two regions with edge degree even. By the Theorem 3.4, the degree of the corresponding vertex in $E_{b}(G)$ becomes odd. By the Theorem2.5, the graph $\mathrm{E}_{\mathrm{b}}(\mathrm{G})$ is non-Eulerian, a contradiction.

Case 2. Assume that the edge lie on only one region with odd degree. By the Theorem 3.4, the degree of the corresponding vertex in $\mathrm{E}_{\mathrm{b}}(\mathrm{G})$ becomes odd. By the Theorem2.5, the graph $\mathrm{E}_{\mathrm{b}}(\mathrm{G})$ is non-Eulerian, a contradiction.

Case 3. Assume that each region contains odd number of edges. By the definition of $\mathrm{E}_{\mathrm{b}}(\mathrm{G})$, each regionvertex is adjacent to all vertices those are the edges covered by the region in G. Since each region contains odd number of edges, the corresponding regionvertex of degree is odd. By the
Theorem2.5, the graph $\mathrm{E}_{\mathrm{b}}(\mathrm{G})$ is non-Eulerian, a contradiction.

Hence $\mathrm{G}$ must satisfies all the above a conditions.

Conversely suppose a graph $G$ satisfies all the conditions. Suppose edgedegree is odd if it lies in two regions. By the Theorem 3.4, the corresponding vertex in $\mathrm{E}_{\mathrm{b}}(\mathrm{G})$ becomes even. Further edgedegree of edge is even if the edge lies on one region. Again by the Theorem 3.4, the degree of the corresponding vertex in $\mathrm{E}_{\mathrm{b}}(\mathrm{G})$ becomes even. Lastly each region contains even number of edges. By the definition of $E_{b}(G)$, the degree of the corresponding vertex is even. Thus each vertex is of degree even Hence $E_{b}(G)$ is Eulerian.

In the following theorem we obtain the condition for the Hamiltonian on Edge semientire block graph of a graph.

Theorem 3.9 For any graph $G, E_{b}(G)$ is always Hamiltonian.

Proof. Suppose $\mathrm{G}$ is any graph. Let $\mathrm{e}_{1}, \mathrm{e}_{2}, \ldots \mathrm{e}_{\mathrm{n}-1}$ be the edge set, $b_{1}, b_{2}, \ldots b_{i}$ be the blocks and $r_{1}, r_{2}, \ldots r_{k}$ be the regions of $G$ such that $\mathrm{e}_{1}, \mathrm{e}_{2}, \ldots \mathrm{e}_{\ell} \in \mathrm{V}\left(\mathrm{b}_{1}\right), \mathrm{e}_{\ell+1}, \mathrm{e}_{\ell+2} \ldots, \ldots \mathrm{e}_{\mathrm{m}} \in \mathrm{V}\left(\mathrm{b}_{2}\right), \ldots$ $\mathrm{e}_{\mathrm{m}+1}, \mathrm{e}_{\mathrm{m}+2}, \ldots \mathrm{e}_{\mathrm{n}-1} \in \mathrm{V}\left(\mathrm{b}_{\mathrm{i}}\right)$. By the Theorem 3.3, V $\left[\mathrm{E}_{\mathrm{b}}(\mathrm{G})\right]=\mathrm{e}_{1}$, $\mathrm{e}_{2}, \ldots \mathrm{e}_{\mathrm{n}-1} \cup \mathrm{b}_{1}, \mathrm{~b}_{2}, \ldots \mathrm{b}_{\mathrm{i}} \cup \mathrm{r}_{1}, \mathrm{r}_{2}, \ldots \mathrm{r}_{\mathrm{k}}$. By theorem $3.2 \mathrm{E}_{\mathrm{b}}(\mathrm{G})$ is always non -separable. By the definition, $b_{1} e_{1}, e_{2}, \ldots e_{\ell-1}$ $r_{1} b_{2} \ldots r_{2} e_{m} b_{3} \ldots e_{k+1}, e_{k+2}, \ldots e_{n-1} b_{k} r_{k} e_{\ell} b_{1}$ form a cycle which contains all the vertices of $E_{b}(G)$. Hence $E_{b}(G)$ is Hamiltonian.

\section{CONCLUSION}

In this paper the relation between the line graph and the edge semientire block graph is introduced. Further the conditions of planarity, Hamiltonian and Eulerian are established.

\section{REFERENCES}

[1] Chartrand G, Geller, D and Hedetniemi, S. J. Combinatorial Theory (1971)10:12.

[2] Harary F., Graph theory, Addition - Weseley Reading .Mass.(1969), pp 34 and 107.

[3] Harary, F., Annals of New York Academy of Science,(1975),175:198.

[4] Kulli V R., On minimally non-outer planar graphs, Proceeding of the Indian National Science Academy, Vol.41, Part A, No.3 (1975), pp 275 -280.

[5] Y.B. Maralabhavi and Venkanagouda. M. Goudar."On Block edge cut vertex graph", in the Journal of ACTA CIENCIA INDICA, Vol. XXXIII M.No.2, (2007) pp. 493-497. 